\begin{tabular}{|c|c|c|}
\hline \multirow{3}{*}{$\begin{array}{r}\text { Case Reports in } \\
\text { Gastroenterology }\end{array}$} & \multirow{2}{*}{\multicolumn{2}{|c|}{ Case Rep Gastroenterol 2014;8:18-25 }} \\
\hline & & \\
\hline & $\begin{array}{l}\text { DOI: 10.1159/000358192 } \\
\text { Published online: January 15, } 2014\end{array}$ & $\begin{array}{l}\text { (c) } 2014 \text { S. Karger AG, Basel } \\
\text { 1662-0631/14/0081-0018 } \$ 39.50 / 0 \\
\text { www.karger.com/crg }\end{array}$ \\
\hline & \multicolumn{2}{|c|}{$\begin{array}{l}\text { This is an Open Access article licensed under the terms of the Creative Common } \\
\text { Attribution-NonCommercial } 3.0 \text { Unported license (CC BY-NC) (www.karger.com/OA } \\
\text { license), applicable to the online version of the article only. Distribution permitted for non } \\
\text { commercial purposes only. }\end{array}$} \\
\hline
\end{tabular}

\title{
The Effects of Endothelial Cells- Preserving Technique on Microsurgical Vascular Reconstruction in Biliary Tract Malignancy: Report of Twenty Cases
}

\author{
Shigehito Miyagi ${ }^{a} \quad$ Wataru Nakanishi ${ }^{a} \quad$ Naoki Kawagishi ${ }^{a}$ \\ Hiroshi Yoshida $^{b}$ Michiaki Unno $^{b}$ Noriaki Ohuchi $^{a}$ \\ ${ }^{a}$ Division of Transplantation, Reconstruction and Endoscopic Surgery and ${ }^{b}$ Division of \\ Digestive Surgery, Tohoku University Graduate School of Medicine, Sendai, Japan
}

\section{Key Words}

Microsurgery $\cdot$ Cholangiocarcinoma $\cdot$ Reconstruction of the hepatic artery $\cdot$ Endothelial cells

\begin{abstract}
We describe our experience of resectional surgery with microsurgical reconstruction of the hepatic arteries in 20 cases with biliary tract malignancy. Hepatic artery thrombosis (HAT) is a lethal complication; therefore, it is important to perform microsurgical reconstruction safely. Recently, we adopted the back wall support suture technique with double needle sutures that does not require the damaged short arteries to be turned over. In this technique, each stitch is placed from the inner side to the outer side to keep endothelial cells. The purpose of this study was to develop safety methods. From 2003 to 2012, 20 patients with biliary tract malignancy with possible involvement of the hepatic arteries underwent resectional surgery with microvascular reconstruction (cholangiocarcinoma: $n=15$; others: $n=5$ ). For this cohort study, patients were divided into two groups: group I $(n=5)$ included patients who underwent the conventional 'twist technique' and group II $(n=15)$ included patients who underwent the microsurgical back wall support suture technique with double needle sutures and received gabexate mesilate, a strong serine protease inhibitor $(40 \mathrm{mg} / \mathrm{kg} /$ day) for 7 days. We investigated HAT using Doppler ultrasonography for 10 days. No postoperative mortality was observed. The incidence of HAT was only one case in group I, and there was no significant difference between the two groups. However, the value of the pulsatile index and acceleration time were significantly improved in group II. In conclusion, the back wall support suture technique with gabexate mesilate administration during microvascular reconstruction
\end{abstract}


Miyagi et al.: The Effects of Endothelial Cells-Preserving Technique on Microsurgical Vascular Reconstruction in Biliary Tract Malignancy: Report of Twenty Cases

was found to be safe. It is important to keep endothelial cells healthy for microvascular reconstruction.

(C) 2014 S. Karger AG, Basel

\section{Introduction}

Microsurgical reconstruction of the hepatic artery is a sometimes challenging technique in cases with biliary tract malignancy because the hepatic artery is located deep within the abdominal cavity, is short and shows very severe intimal damage because of lymph node dissection $[1,2]$, especially in cases with hilar cholangiocarcinoma, where the intimal damage is very severe because of tumor invasion. Furthermore, hepatic artery thrombosis (HAT) is one of the lethal complications of this procedure. Thus, performing the conventional reconstruction 'twist technique' may not be safe, as it involves turning over the damaged short arteries (fig. 1), which may result in separation of the endothelial lining from the vascular wall $[1,2]$. It is one of the most important things to develop a safe method for microvascular reconstruction. Once the new method is developed, the indication of surgery for biliary tract might change widely. We inferred that the key to successful microvascular reconstruction is to preserve the endothelial cells.

To address this, we adopted the back wall support suture technique with double needle sutures (fig. 1) which does not require the damaged short arteries to be turned over. Briefly, we placed two sutures at the deepest, most difficult points in the artery for backside support. Each stitch was placed from the inner side of the arterial wall to the outer side, with double needle sutures for safe intimal adaptation [3]. The subsequent sutures were placed in front and on either side, adjacent to the previous sutures.

The patients also received systemic administration of gabexate mesilate, a strong serine protease inhibitor [4,5]. This drug has been shown to be more effective in the treatment of disseminated intravascular coagulation as compared to heparin [4], since it shows a protective effect on endothelial cells. In fact, we have previously reported that serine protease inhibitors have cytoprotective effects on endothelial cells in ischemia-reperfusion injury [5]. Herein we describe our experience of resectional surgery with microsurgical reconstruction of the hepatic artery in 20 cases with biliary tract malignancy. The purpose of this study was to examine the effects of our methods (microsurgical reconstruction with double needle sutures and systemic administration of gabexate mesilate) and to reveal the significance of endothelial cells for microvascular reconstruction. This study is the first to report the significance of endothelial cells and the safety of this procedure for biliary tract malignancy.

\section{Materials and Methods}

All procedures were reviewed and approved by the Ethical Committee of Tohoku University School of Medicine and were performed in accordance with the ethical standards of the Declaration of Helsinki.

From September 2003 to December 2012, 20 patients with biliary tract malignancy along with possible involvement of the hepatic artery underwent resectional surgery with reconstruction of the hepatic artery (average age $64.5 \pm 11.0$ years; male:female ratio 14:6). The malignancies included cholangiocarcinoma $(n=15)$, gallbladder carcinoma $(n=1)$ and pancreatic cancer $(n=4)$. The surgical procedures carried out included left trisectionectomy $(\mathrm{n}=1)$, left hepatectomy (including extended left hepatectomy) $(n=7)$, right hepatectomy $(n=1)$, central bisectionectomy $(n=2)$, partial resection of segment 5 and inferior area of 
Miyagi et al.: The Effects of Endothelial Cells-Preserving Technique on Microsurgical Vascular Reconstruction in Biliary Tract Malignancy: Report of Twenty Cases

segment $4(n=1)$ and pancreatoduodenectomy $(n=8)$. Reconstruction of the hepatic artery was performed by end-to-end anastomosis using microsurgical techniques (right, $\mathrm{n}=13$; left, $\mathrm{n}=2$; proper, $\mathrm{n}=3$; and posterior, $\mathrm{n}=2$ ).

The patients were divided into two groups: group I $(n=5)$, which included patients who underwent the conventional 'twist technique' between 2003 and 2004 and received heparin anticoagulant therapy on the day of the operation, and group II $(n=15)$, which included patients who underwent the microsurgical back wall support suture technique with double needle sutures between 2005 and 2012 and received gabexate mesilate for 7 days (40 mg/kg/day). We investigated HAT using Doppler ultrasonography for 10 days after reconstruction and examined the pulsatile index (PI) (peak systolic-end diastolic/mean velocities), the resistive index (RI) (peak systolic-end diastolic/peak systolic velocities) and the acceleration time, for the purpose of revealing the condition of endothelial cells [6]. We defined HAT as the absence of hepatic arterial flow as determined by ultrasonography, and worsening of laboratory parameters such as alanine aminotransferase and aspartate aminotransferase levels. On the other hand, although there is no direct relationship between the incidence of HAT and long-term survival rates, we also investigated those rates at 1 and 3 years for the purpose of revealing the significance of those resectional surgeries with reconstruction of hepatic arteries.

Patients were observed from the date of operation until death or the last follow-up. The values are shown as mean \pm standard deviation. Student's $t$ test was used to compare the categorical data. Kaplan-Meier survival curves were constructed for both study groups. The effects of the treatment on overall survival were initially examined using the Kaplan-Meier log-rank test (Breslow-Gehan-Wilcoxon test). All calculations were made with the JMP Pro software package (SAS Institute, Cary, N.C., USA). A p value $<0.05$ was considered significant.

\section{Results}

The background data of the patients are presented in table 1. It reveals a heterogeneous background, but there were no significant differences between the two groups.

No postoperative mortality or bleeding was observed. HAT occurred in $5.0 \%$ of the patients $(1 / 20)$. The incidence of HAT was lower in group II $(0 \%, 0 / 15)$ than in group I $(20.0 \%, 1 / 5)$, but there was no significant difference between two groups $(\mathrm{p}=0.25$, Student's t test). In the case with HAT (case 3, the posterior branch reconstruction case), rereconstruction could not be performed due to a very short arterial neck and severe intimal damage. Thus, arterioportal shunting was performed, and the patient recovered. In the HAT case, the PI value decreased to $<0.4$, with a delay in acceleration time to $>0.1 \mathrm{~s}$ during the first reconstruction of the hepatic artery, whereas the other cases had PI values $>0.5$ and acceleration times of $<0.1 \mathrm{~s}$ for 7 days after operation.

On 7 days after operation, the RI value was higher in group II than in group I, but there was no significant difference between the two groups ( $p=0.17$, Student's $t$ test). On the other hand, the PI value was significantly higher in group II than in group I ( $<<0.05)$. Furthermore, acceleration time was significantly shorter in group II than in group I ( $p<$ 0.05). From these findings, we could understand that the condition of endothelial cells had improved better in group II (table 2). On the other hand, on day 0 after operation, there were no significant differences between the two groups except for acceleration time.

During the course of this study, 10 patients died due to recurrence of their original disease. In all cases, the surgical margin was negative, except for 2 cases in group II. 
Miyagi et al.: The Effects of Endothelial Cells-Preserving Technique on Microsurgical Vascular Reconstruction in Biliary Tract Malignancy: Report of Twenty Cases

The overall survival rates of the biliary tract malignancy cohort at 1 and 3 years were 59.8 and $23.6 \%$, respectively (fig. 2). In groups I and II, the survival rates at 3 years were 0 and $59.1 \%$, respectively. There were no significant differences in the survival rate between the two groups ( $p=0.06$, Kaplan-Meier log-rank test), possibly due to the limited number of cases (fig. 2). In cases with cholangiocarcinoma, the survival rates at 1 and 3 years were 64.6 and $25.1 \%$, respectively. On the other hand, in cases with gallbladder carcinoma and pancreatic cancer, the survival rate at 1 year was 33.3\%. There were no significant differences in the survival rate between the two groups (Kaplan-Meier log-rank test) (fig. 2).

In cases with histopathological arterial involvement $(n=11)$, the survival rate at 1 and 2 years was 45.4 and $27.3 \%$, respectively. On the other hand, in those without histopathological arterial involvement $(\mathrm{n}=9)$, the survival rates at 1,2 and 3 years were 83.3, 83.3 and $41.7 \%$, respectively (fig. 2). There were significant differences in the survival rate between groups I and II ( $p=0.03$, Kaplan-Meier log-rank test).

\section{Discussion}

Our results show that the acceleration times after operation were significantly short in group II by ultrasonography examination. Furthermore, the PI value in group II was also significantly higher than that in group I on day 7 after operation. This means that there was no stenosis at the reconstructed artery and that the inflow blood volume was good until day 7 after operation. These results suggest that the back wall support suture technique with double needle sutures is a safe technique for preserving arterial intimae and endothelial cells. On the other hand, RI values were similar between the two groups in this study. The RI value was strongly influenced by end diastolic velocity and outflow volume rather than by peak systolic velocity and inflow volume. Thus, if the portal flow was not so good, the end diastolic velocity of the hepatic artery became high and the RI value became low. We think this is the reason why the values were similar between the two groups in our study.

Conventional plastic surgical reconstruction using the 'twist technique', which involves turning over the damaged short arteries, may not be safe, as it often results in separation of the intimae from the vascular wall and is not always possible in short branch reconstruction cases. Thus, we adopted the back wall support suture technique from 2005 to improve safety. Although this procedure was complicated, it was useful, particularly in cases with damaged short arteries. It should be noted that our study included a small number of patients, but we successfully reconstructed the hepatic artery in our biliary malignancy patients and developed a safe method for microvascular reconstruction using this new procedure and gabexate mesilate. There have been few articles so far describing the risk of the conventional technique and the significance of safety reconstruction. The most important conclusion we want to draw from this study is not to use our method, but to keep endothelial cells healthy on microvascular reconstruction.

In Japan, gabexate mesilate is sometimes used in the treatment of disseminated intravascular coagulation and has been shown to be more effective than heparin in some cases. Gabexate mesilate is one of the strongest serine protease inhibitors. When using serine protease inhibitors in marginal donor liver transplantation of rats [5], we discovered their cytoprotective effects on endothelial cells. We believe that the key to successful microvascular reconstruction is preservation of endothelial cells. Thus, in this study we investigated the effects of gabexate mesilate $(40 \mathrm{mg} / \mathrm{kg})$ along with the use of the back wall support suture technique for microvascular reconstruction. There were no cases of HAT or postoperative 
Miyagi et al.: The Effects of Endothelial Cells-Preserving Technique on Microsurgical Vascular Reconstruction in Biliary Tract Malignancy: Report of Twenty Cases

bleeding in group II owing to the anticoagulant effects of gabexate mesilate, which is a significant finding.

It is known that the repair of the intimae on the reconstructed artery is completed within 6-10 days after surgery, and stenosis also occurs within the same time frame $[2,3,6]$. HAT has also been reported to occur within 6-10 days after surgery. We evaluated the wave shape of the hepatic artery and the acceleration time via ultrasonography in cases of microsurgical reconstruction in liver transplantation, and we observed that wave shape changes were often seen 5-7 days after operation. Thus, we administered gabexate mesilate to the patients in group II for at least 7 days in order to keep the endothelial cells intact, and performed ultrasonography 10 days after operation. We did not administer heparin to the group II patients because of our previous experience of major bleeding in a few liver transplantation cases who had received heparin. As a result, there were no cases of HAT and postoperative bleeding in group II.

The surgical indications for reconstruction of the hepatic artery in cases of biliary malignancy remain controversial. Shimada et al. [7], based on their experience of 12 patients who underwent hepatic arterial reconstruction, reported that reconstruction of the hepatic artery cannot be recommended for gallbladder cancer patients due to their poor prognosis. Our results also indicate that the survival rate of patients with cholangiocarcinoma who underwent arterial reconstruction might be better than that of those with gallbladder cancer and pancreatic cancer.

It remains to be determined whether arterial reconstruction in cases of biliary malignancy prolongs patient survival. Our sample size was insufficient to address this issue. Shimada et al. [7] and Kaneoka et al. [8] reported some cases who underwent arterial reconstruction for hilar bile duct cancer and survived for more than 3 years post surgery. Nagino et al. [9] reported 50 patients with hilar cholangiocarcinoma in whom the hepatic arteries were reconstructed. They reported improved survival in cases who received resectional surgery, but also reported difficulties performing that procedure safely and the usefulness of portal vein arterialization $[9,10]$. Thus, safer reconstruction techniques need to be developed.

In conclusion, the back wall support suture technique with double needle sutures for microvascular reconstruction along with administration of gabexate mesilate was found to be safe and effective in cases of biliary tract malignancy. Thus, it is possible to safely perform resectional surgery in biliary tract malignancy cases with hepatic artery involvement. It is important to keep endothelial cells healthy for microvascular reconstruction.

\section{Acknowledgements}

This work was supported by Grants-in-Aid for Scientific Research from the Ministry of Education, Science, and Culture of Japan and from the Ministry of Welfare of Japan, and by a grant from Tohoku University Graduate School of Medicine. The authors thank Yu Katayose, associate professor at Tohoku University, and Toshiki Rikiyama, professor at Jichi Medical University, for support.

\section{Disclosure Statement}

The authors have no conflict of interest. 


\begin{tabular}{l|l}
\hline Case Rep Gastroenterol 2014;8:18-25 \\
\hline DOI: 10.1159/000358192 & $\begin{array}{l}\text { C 2014 S. Karger AG, Basel } \\
\text { www.karger.com/crg }\end{array}$ \\
\hline
\end{tabular}

Miyagi et al:: The Effects of Endothelial Cells-Preserving Technique on Microsurgical Vascular Reconstruction in Biliary Tract Malignancy: Report of Twenty Cases

\section{References}

1 Sakamoto Y, Sano T, Shimada K, Kosuge T, Kimata Y, Sakuraba M, Yamamoto J, Ojima H: Clinical significance of reconstruction of the right hepatic artery for biliary malignancy. Langenbecks Arch Surg 2006;391:203208.

-2 Shirouzu Y, Kasahara M, Morioka D, Sakamoto S, Taira K, Uryuhara K, Ogawa K, Takada Y, Egawa H, Tanaka K: Vascular reconstruction and complications in living donor liver transplantation in infants weighing less than 6 kilograms: the Kyoto experience. Liver Transpl 2006;12:1224-1232.

-3 Miyagi S, Enomoto Y, Sekiguchi S, Kawagishi N, Sato A, Fujimori K, Satomi S: Microsurgical back wall support suture technique with double needle sutures on hepatic artery reconstruction in living-donor liver transplantation. Transplant Proc 2008;40:2521-2522.

4 Mikami K, Goto T, Miura K, Ohshima S, Yoneyama K, Lin JG, Watanabe D, Segawa D, Kataoka E, Shibuya T, Watanabe S: Gabexate mesilate, a synthetic protease inhibitor, attenuates carbon tetrachloride-induced liver injury in rats. J Gastroenterol 2005;40:260-265.

5 Miyagi S, Ohkohchi N, Oikawa K, Satoh M, Tsukamoto S, Satomi S: Effects of anti-inflammatory cytokine agent (FR167653) and serine protease inhibitor on warm ischemia-reperfusion injury of the liver graft. Transplantation 2004;77:1487-1493.

-6 Kaneko J, Sugawara Y, Akamatsu N, Kishi Y, Niiya T, Kokudo N, Makuuchi M, Mizuta K: Prediction of hepatic artery thrombosis by protocol Doppler ultrasonography in pediatric living donor liver transplantation. Abdom Imaging 2004;29:603-605.

7 Shimada H, Endo I, Sugita M, Masunari H, Fujii Y, Tanaka K, Misuta K, Sekido H, Togo S: Hepatic resection combined with portal vein or hepatic artery reconstruction for advanced carcinoma of the hilar bile duct and gallbladder. World J Surg 2003;27:1137-1142.

8 Kaneoka Y, Yamaguchi A, Isogai M, Suzuki M: Longer than 3-year survival following hepato-ligamentopancreatoduodenectomy for hilar cholangiocarcinoma with vascular involvement: report of a case. Surg Today 2003;33:772-776.

-9 Nagino M, Nimura Y, Nishio H, Ebata T, Igami T, Matsushita M, Nishikimi N, Kamei Y: Hepatectomy with simultaneous resection of the portal vein and hepatic artery for advanced perihilar cholangiocarcinoma: an audit of 50 consecutive cases. Ann Surg 2010;252:115-123.

10 Hirano S, Kondo S, Tanaka E, Shichinohe T, Tsuchikawa T, Kato K, Matsumoto J, Kawasaki R: Outcome of surgical treatment of hilar cholangiocarcinoma: a special reference to postoperative morbidity and mortality. J Hepatobiliary Pancreat Sci 2010;17:455-462.

Table 1. Background of the patients

\begin{tabular}{lll}
\hline & Group I & Group II \\
\hline Male:female ratio & $2: 3$ & $12: 3$ \\
Age, years & $56.8 \pm 12.3$ & $67.1 \pm 9.6$ \\
Height, cm & $161.2 \pm 7.5$ & $159.7 \pm 7.4$ \\
Weight, kg & $60.4 \pm 5.3$ & $55.0 \pm 11.4$ \\
Cholangiocarcinoma (including ICC) & 3 & 12 \\
Gallbladder carcinoma & 1 & 0 \\
Pancreatic cancer & 1 & 3 \\
pA(+) & 3 & 8 \\
Artery reconstruction, rt/lt/post/PHA & $2 / 1 / 1 / 1$ & $11 / 1 / 1 / 2$ \\
\hline
\end{tabular}

ICC = Intrahepatic cholangiocarcinoma; lt = left hepatic artery; $\mathrm{pA}(+)=$ pathological arterial involvement; $\mathrm{PHA}=$ proper hepatic artery; post = posterior branch of the right hepatic artery; rt = right hepatic artery. 
Miyagi et al.: The Effects of Endothelial Cells-Preserving Technique on Microsurgical Vascular Reconstruction in Biliary Tract Malignancy: Report of Twenty Cases

Table 2. Results of ultrasonography examinations

\begin{tabular}{lllc}
\hline & Group I & Group II & p value \\
\hline HAT & $1 / 5$ & $0 / 15$ & 0.250 \\
PI POD 0 & $0.690 \pm 0.154$ & $0.869 \pm 0.179$ & 0.121 \\
RI POD 0 & $0.464 \pm 0.082$ & $0.529 \pm 0.054$ & 0.058 \\
Acceleration time POD 0, s & $0.089 \pm 0.038$ & $0.037 \pm 0.007$ & $<0.001^{*}$ \\
PI POD 7 & $0.663 \pm 0.066$ & $0.785 \pm 0.089$ & $0.020^{*}$ \\
RI POD 7 & $0.445 \pm 0.093$ & $0.505 \pm 0.069$ & 0.170 \\
Acceleration time POD 7, s & $0.084 \pm 0.008$ & $0.037 \pm 0.008$ & $<0.001^{*}$ \\
\hline
\end{tabular}

POD = Postoperative day. ${ }^{*}$ Statistically significant.
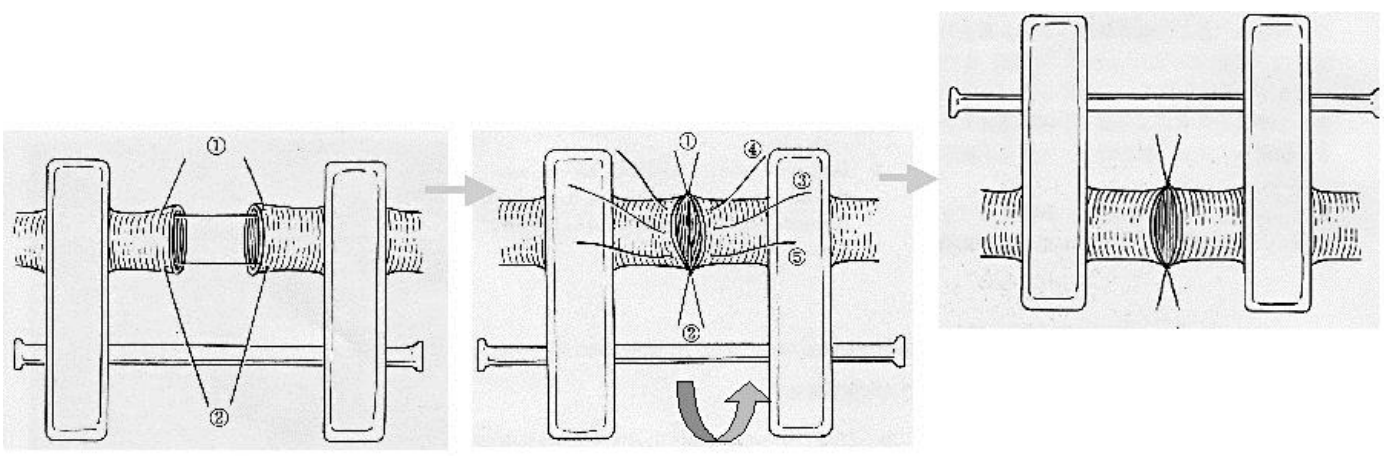

\section{We need to turn over the hepatic artery.}
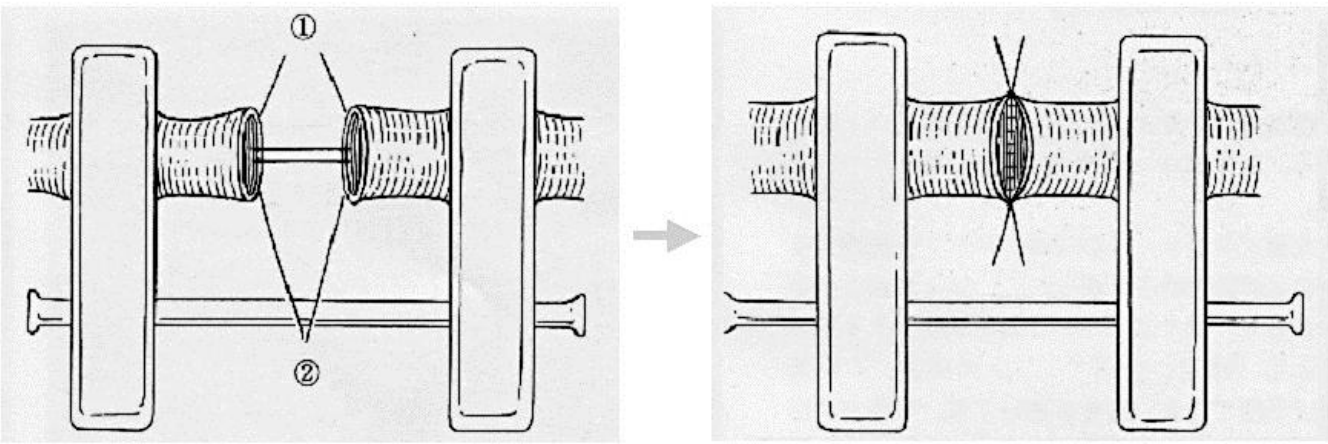

We don't need to turn over the hepatic artery!

Fig. 1. Conventional 'twist technique' and back wall support suture technique with double needle sutures. 
Miyagi et al.: The Effects of Endothelial Cells-Preserving Technique on Microsurgical Vascular Reconstruction in Biliary Tract Malignancy: Report of Twenty Cases

\section{survival rate}
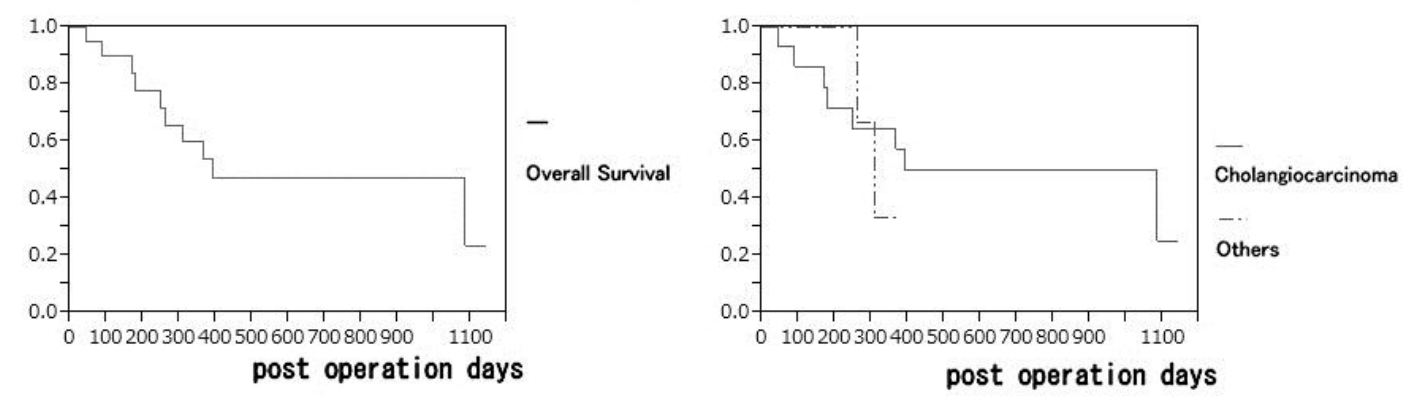

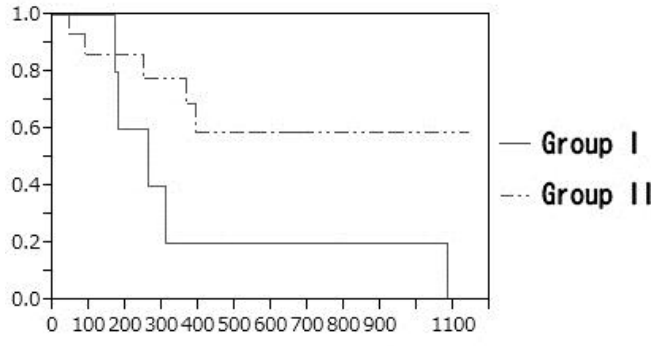

post operation days

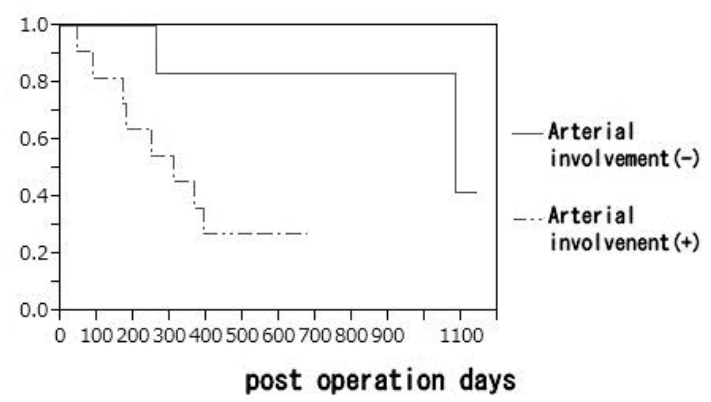

Fig. 2. Survival rates. Group I: patients who underwent the conventional 'twist technique' and received heparin anticoagulant therapy. Group II: patients who underwent the microsurgical back wall support suture technique and received gabexate mesilate therapy $(40 \mathrm{mg} / \mathrm{kg} /$ day) for 7 days. Cholangiocarcinoma: patients with cholangiocarcinoma. Others: patients with gallbladder carcinoma and pancreatic cancer. Arterial involvement(-): patients without pathological arterial involvement. Arterial involvement $(+)$ : patients with pathological arterial involvement. 